VoL. 66 (2002) [95-104]

\title{
POSITIVE SOLUTIONS OF FOURTH-ORDER SUPERLINEAR SINGULAR BOUNDARY VALUE PROBLEMS
}

\author{
Guoliang Shi and Shaozhu Chen
}

This paper investigates fourth-order superlinear singular two-point boundary value problems and obtains necessary and sufficient conditions for existence of $C^{2}$ or $C^{3}$ positive solutions on the closed interval.

\section{INTRODUCTION}

In this paper, we are concerned with the fourth-order singular two-point boundary value problem

$$
\begin{cases}u^{(4)}(t)=f(t, u(t)), & 0<t<1, \\ u(0)=u(1)=u^{\prime \prime}(0)=u^{\prime \prime}(1)=0, & \end{cases}
$$

where $f \in C((0,1) \times[0,+\infty),[0,+\infty))$ and is quasi-homogeneous with respect to the second variable, namely, there are constants $\lambda, \mu, N, M$ with $1<\lambda \leqslant \mu<\infty$ and $0<N \leqslant 1 \leqslant M$ such that for all $0<t<1, u \geqslant 0$,

$$
\begin{array}{ll}
c^{\mu} f(t, u) \leqslant f(t, c u) \leq c^{\lambda} f(t, u), & \text { if } \quad 0<c \leqslant N, \\
c^{\lambda} f(t, u) \leqslant f(t, c u) \leq c^{\mu} f(t, u), & \text { if } \quad c \geqslant M .
\end{array}
$$

A typical quasi-homogeneous function is $f=f_{1}(t) u^{\lambda_{1}}+\cdots+f_{m}(t) u^{\lambda_{m}}$, where $\lambda$ $\leqslant \lambda_{i} \leqslant \mu, i=1, \ldots, m$.

Singular or nonsingular fourth-order boundary value problems have been extensively studied by many authors (see $[\mathbf{1}, \mathbf{2}, \mathbf{3}, \mathbf{4}, \mathbf{5}, \mathbf{6}, \mathbf{7}]$ for nonsingular cases and $[\mathbf{8}, \mathbf{9}]$ for singular cases). In $[\mathbf{3}, \mathbf{4}, \mathbf{5}]$ the right hand side function in the equation of (1) has separated variables, namely, $f(t, u)=\lambda a(t) g(u)$, and in $[\mathbf{1}, \mathbf{6}, \mathbf{7}, \mathbf{8}]$ the function $f$ involves the second derivative $u^{\prime \prime}$. O'Regan considered the singular case where $f\left(t, u, u^{\prime \prime}\right)$ is singular at $u=0$ or $u^{\prime \prime}=0$, while in [9] singularity occurs at $t=0$ or $t=1$. Using a modified upper and lower solution method, Chen and Zhang [10] established necessary and sufficient conditions for existence of positive solutions to second-order sublinear

Received 8th January, 2002

This research was supported by the Chinese NSF under Grant 10071043

Copyright Clearance Centre, Inc. Serial-fee code: 0004-9727/02 \$A2.00+0.00. 
boundary value problems on a half-line. Using a similar method, Wei [9] obtained necessary and sufficient conditions for existence of positive solutions to the fourth-order problem (1) in the sublinear case. The results in $[9, \mathbf{1 0}]$ involve integrability conditions in terms of the function $f$ and the Green's function. To this connection, however, the upper and lower solution method can hardly be used to treat the superlinear case.

In this paper, based on a careful analysis of the Green's function, we shall apply a fixed point theorem in cones to the superlinear problem (1) and obtain necessary and sufficient conditions for existence of a positive solution with different smoothness on the closed interval.

\section{Main Results}

Our main results are the two following theorems.

THEOREM 1. The boundary value problem (1) has a positive solution $u$ $\in C^{2}[0,1] \cap C^{4}(0,1)$, if and only if,

$$
\int_{0}^{1} t(1-t) f(t, t(1-t)) d t<\infty
$$

THEOREM 2. The boundary value problem (1) has a positive solution u $\in C^{3}[0,1] \cap C^{4}(0,1)$, if and only if,

$$
\int_{0}^{1} f(t, t(1-t)) d t<\infty
$$

We note that (5) implies (4). To prove Theorems 1 and 2, we shall prepare some lemmas. First, we state a fixed point theorem in a cone as follows:

Lemma 1. ([11, Theorem 2.3.4].) Let $E$ be a Banach space and $P$ a cone in $E$. Suppose that $\Omega_{1}$ and $\Omega_{2}$ are two bounded open subsets of $E$ with $\theta \in \Omega_{1}, \bar{\Omega}_{1} \subset \Omega_{2}$. If $T: P \cap\left(\bar{\Omega}_{2} \backslash \Omega_{1}\right) \rightarrow P$ is a completely continuous operator satisfying

$$
\|T x\| \leqslant\|x\| \text { for } x \in P \cap \partial \Omega_{1} \text { and }\|T x\| \geqslant\|x\| \text { for } x \in P \cap \partial \Omega_{2},
$$

then $T$ has a fixed point in $P \cap\left(\bar{\Omega}_{2} \backslash \Omega_{1}\right)$.

Let $E=\left\{u \in C^{2}[0,1]: u(0)=u(1)=0, u^{\prime \prime}(0)=u^{\prime \prime}(1)=0\right\}$. Define the norm $\|u\|$ for every $u \in E$ by $\|u\|=|u|_{0}+\left|u^{\prime \prime}\right|_{0}$, where $|\cdot|_{0}$ is the usual sup-norm for continuous functions over $[0,1]$. It is seen that $E$ equipped with the norm $\|\cdot\|$ is a Banach space.

Let $G(t, s)$ be the Green's function of the second-order boundary value problem

$$
\left\{\begin{array}{l}
-u^{\prime \prime}(t)=0, \\
u(0)=u(1)=0,
\end{array}\right.
$$


that is,

$$
G(t, s)= \begin{cases}s(1-t), & 0 \leqslant s \leqslant t \leqslant 1 \\ t(1-s), & 0 \leqslant t \leqslant s \leqslant 1\end{cases}
$$

Let

$$
h(t, s)=\int_{0}^{1} G(t, \tau) G(\tau, s) d \tau
$$

Then $h(t, s)$ is the Green's function of the homogeneous fourth-order boundary value problem corresponding to (1). It is easily seen that

$$
G(t, s) \leqslant G(s, s), \quad 0 \leqslant t, s \leqslant 1
$$

and for $1 / 4 \leqslant t \leqslant 3 / 4$,

$$
G(t, s) \geqslant \frac{1}{4} G(s, s), \quad 0 \leqslant s \leqslant 1 .
$$

Denote

$$
\begin{aligned}
P=\left\{u \in E \mid u(t) \geqslant 0, u^{\prime \prime}(t) \leqslant 0,0\right. & \leqslant t \leqslant 1 ; \\
& \left.u(t) \geqslant \frac{1}{4}|u|_{0},-u^{\prime \prime}(t) \geqslant \frac{1}{4}\left|u^{\prime \prime}\right|_{0}, \frac{1}{4} \leqslant t \leqslant \frac{3}{4}\right\} .
\end{aligned}
$$

It can be easily seen that $P$ is a cone in $E$.

Next, we define an operator $T: P \rightarrow E$ by

$$
(T u)(t)=\int_{0}^{1} h(t, s) f(s, u(s)) d s, \quad u \in P .
$$

We observe that a fixed point of $T$ in $E$ is indeed a positive solution of the boundary value problem (1).

Using the Green's function, for every $u \in P$, we shall have an estimate for $u(t)$ in terms of the magnitude of its second derivative, namely, for $t \in[0,1]$,

$$
\begin{aligned}
u(t) & =\int_{0}^{1} G(t, s)\left(-u^{\prime \prime}(s)\right) d s \leqslant\left(\int_{0}^{t} s(1-t) d s+\int_{t}^{1} t(1-s) d s\right)\left|u^{\prime \prime}\right|_{0} \\
& =\frac{1}{2} t(1-t)\left|u^{\prime \prime}\right|_{0} .
\end{aligned}
$$

Let $u \in P$ and let $c$ be a positive number such that $c \geqslant M$ and $\left|u^{\prime \prime}\right|_{0} /(2 c) \leqslant N$. From (9), $u(s) /(c s(1-s)) \leqslant\left|u^{\prime \prime}\right|_{0} /(2 c) \leqslant M$. Then, from (2) and (3),

$$
\begin{aligned}
|T u(t)| & \leqslant \int_{0}^{1} G(s, s) c^{\mu} f(s, u(s) / c) d s \\
& =c^{\mu} \int_{0}^{1} G(s, s) f\left(s, \frac{u(s)}{c s(1-s)} s(1-s)\right) d s \\
& \leqslant c^{\mu}\left(\frac{\left|u^{\prime \prime}\right|_{0}}{2 c}\right)^{\lambda} \int_{0}^{1} s(1-s) f(s, s(1-s)) d s .
\end{aligned}
$$

Hence, $T$ is well defined on $P$ provided that (4) or (5) holds. 
Lemma 2. If (4) holds, then $T(P) \subset P$.

Proof: Let $u \in P$. Obviously, $T u(t) \geqslant 0$ and $-(T u)^{\prime \prime}(t) \geqslant 0$. For $1 / 4 \leqslant t$ $\leqslant 3 / 4$, we claim that

$$
T u(t) \geqslant \frac{1}{4}|T u|_{0}
$$

Indeed, from (4), by Fubini's theorem, (8) can be rewritten as

$$
(T u)(t)=\int_{0}^{1} G(t, \tau) \int_{0}^{1} G(\tau, s) f(s, u(s)) d s d \tau .
$$

It follows from (6) that

$$
|T u|_{0} \leqslant \int_{0}^{1} G(\tau, \tau) \int_{0}^{1} G(\tau, s) f(s, u(s)) d s d \tau .
$$

On the other hand, for $1 / 4 \leqslant t \leqslant 3 / 4,(7)$ together with (11) gives

$$
(T u)(t) \geqslant \frac{1}{4} \int_{0}^{1} G(\tau, \tau) \int_{0}^{1} G(\tau, s) f(s, u(s)) d s d \tau \geq \frac{1}{4}|T u|_{0} .
$$

Next, we claim that $-(T u)^{\prime \prime}(t) \geqslant(1 / 4)\left|(T u)^{\prime \prime}\right|_{0}$ for $t \in[1 / 4,3 / 4]$. In fact, from

$$
-(T u)^{\prime \prime}(t)=\int_{0}^{1} G(t, s) f(s, u(s)) d s
$$

it follows from (6) and (7) that

$$
\left|(T u)^{\prime \prime}\right|_{0} \leq \int_{0}^{1} G(s, s) f(s, u(s)) d s
$$

and, for $1 / 4 \leqslant t \leqslant 3 / 4$,

$$
-(T u)^{\prime \prime}(t) \geqslant \frac{1}{4} \int_{0}^{1} G(s, s) f(s, u(s)) d s \geqslant \frac{1}{4}\left|(T u)^{\prime \prime}\right|_{0} .
$$

We now conclude that $T: P \rightarrow P$ from (12) and (13) and complete the proof.

Lemma 3. If (4) holds, then $T$ is a completely continuous operator on $P$.

Proof: If $u_{n} \in P$ and $u_{n} \rightarrow u_{0}$ in $E$ as $n \rightarrow \infty$, then we have that $\dot{u}_{0} \in P$ by the definition of the cone $P$ and that $\left\{\left\|u_{n}\right\|\right\}$ is bounded, say, $\left\|u_{n}\right\| \leqslant C_{0}, n \geqslant 1$. As a result, from (9), we have

$$
u_{n}(t) \leqslant \frac{C_{0}}{2} t(1-t)
$$


Let $c$ be a positive number such that $c \geqslant M$ and $C_{0} / 2 c \leqslant N$. From (2) and (3),

$$
\begin{aligned}
\left|\left(T u_{n}\right)(t)\right| & \leqslant \int_{0}^{1} s(1-s) f\left(s, u_{n}(s)\right) d s \\
& \leqslant \int_{0}^{1} s(1-s) c^{\mu} f\left(s, u_{n}(s) / c\right) d s \\
& \leqslant \int_{0}^{1} s(1-s) c^{\mu}\left(\frac{u_{n}(s)}{s(1-s) c}\right)^{\lambda} f(s, s(1-s)) d s \\
& \leqslant c^{\mu-\lambda}\left(\frac{C_{0}}{2}\right)^{\lambda} \int_{0}^{1} s(1-s) f(s, s(1-s)) d s .
\end{aligned}
$$

Now, from (4), an application of Lebesgue's dominant convergence theorem gives the continuity of $T$ on $P$.

To prove $T$ is a compact operator, we shall show that for every bounded sequence $\left\{u_{n}\right\}$ in $P$, the sequence $\left\{T u_{n}\right\} \subset P$ has a convergent subsequence in $E$. Since $\left\{T u_{n}\right\}$ is bounded in $E,\left\{\left|\left(T u_{n}\right)^{\prime \prime}\right|_{0}\right\}$ is bounded and hence $\left\{T u_{n}(t)\right\}$ is equicontinuous. By Ascoli-Arzela's lemma, it suffices to show that $\left\{\left(T u_{n}\right)^{\prime \prime}(t)\right\}$ is equicontinuous. Let $C_{0}$ be a positive number such that $\left\|u_{n}\right\| \leqslant C_{0}, n=1,2, \ldots$ Then (14) holds from (9). Again, choose a $c \geqslant \max \left\{M, C_{0} /(2 N)\right\}$. Then

$$
\begin{aligned}
(T u)^{\prime \prime \prime}(t) & =\int_{0}^{t} s f(s, u(s)) d s-\int_{t}^{1}(1-s) f(s, u(s)) d s \\
& \leqslant \int_{0}^{t} s f(s, u(s)) d s+\int_{t}^{1}(1-s) f(s, u(s)) d s \\
& \leqslant C_{1}\left(\int_{0}^{t} s f(s, s(1-s)) d s+\int_{t}^{1}(1-s) f(s, s(1-s)) d s\right) \\
& =: F(t)
\end{aligned}
$$

where $C_{1}=c^{\mu-\lambda}\left(C_{0} / 2\right)^{\lambda}$. Since, in view of (4),

$$
\begin{aligned}
\int_{0}^{1} F(t) d t & =C_{1} \int_{0}^{1} \int_{0}^{t} s f(s, u(s)) d s d t+C_{1} \int_{0}^{1} \int_{t}^{1}(1-s) f(s, u(s)) d s \\
& =2 C_{1} \int_{0}^{1} s(1-s) f(s, s(1-s)) d s<\infty
\end{aligned}
$$

we have the equicontinuity of the sequence $\left\{\left(T u_{n}\right)^{\prime \prime}(t)\right\}$ from the uniform continuity of the convergent integral of $F(t)$ with respect to the Lebesgue measure over $[0,1]$.

Therefore, $T$ is a compact operator on $P$ and the proof of Lemma 3 is complete.

We are now in a position to prove our main results. 
Proof of Theorem 1: Necessity. Let $u \in C^{2}[0,1] \cap C^{4}(0,1)$ be a positive solution of (1). Obviously, $u^{\prime \prime}(t) \leqslant 0$ for $0 \leqslant t \leqslant 1$ and hence $u(t)$ is concave. It follows from $u(0)=u(1)=0$ that $u^{\prime}(0)>0$ and $u^{\prime}(1)<0$. Consequently, there must be a positive number $k$ such that $u(t) \geqslant k t(1-t)$. Let $c \geqslant \max \{M, 1 /(k N)\}$. Then, for $0<t<1, t(1-t) /(c u(t))<N$, and we get

$$
\begin{aligned}
f(t, t(1-t)) & \leqslant c^{\mu} f(t, t(1-t) u(t) /(c u(t))) \\
& \leqslant c^{\mu-\lambda} k^{-\lambda} f(t, u(t))=c^{\mu-\lambda} k^{-\lambda} u^{(4)}(t) .
\end{aligned}
$$

Since $u^{\prime \prime}(0)=u^{\prime \prime}(1)=0$, there is a $t_{0} \in(0,1)$ such that $u^{\prime \prime \prime}\left(t_{0}\right)=0$. Then

$$
u^{\prime \prime}\left(t_{0}\right)=\int_{0}^{t_{0}} u^{\prime \prime \prime}(s) d s=-\int_{0}^{t_{0}} \int_{s}^{t_{0}} u^{(4)}(\tau) d \tau d s=-\int_{0}^{t_{0}} \tau u^{(4)}(\tau) d \tau
$$

On the other hand,

$$
\begin{aligned}
u^{\prime \prime}\left(t_{0}\right) & =-\int_{t_{0}}^{1} u^{\prime \prime \prime}(s) d s=-\int_{t_{0}}^{1} \int_{t_{0}}^{s} u^{(4)}(\tau) d \tau d s \\
& =-\int_{t_{0}}^{1}(1-\tau) u^{(4)}(\tau) d \tau
\end{aligned}
$$

Therefore,

$$
\begin{aligned}
\int_{0}^{1} t(1-t) u^{(4)}(t) d t & =\left(\int_{0}^{t_{0}}+\int_{t_{0}}^{1}\right) t(1-t) u^{(4)}(t) d t \\
& \leqslant \int_{0}^{t_{0}} t u^{(4)}(t) d t+\int_{t_{0}}^{1}(1-t) u^{4}(t) d t \\
& =2\left(-u^{\prime \prime}\left(t_{0}\right)\right)<\infty
\end{aligned}
$$

We now obtain (4) from (15) and (18), and complete the proof of the necessity.

Sufficiency. Let $\Omega_{1}=\{u \in E \mid\|u\|<r\}$, where

$$
r \leqslant \min \left\{2 N, 2\left(\int_{0}^{1} s(1-s) f(s, s(1-s)) d s\right)^{1 /(1-\lambda)}\right\} .
$$

Let $u \in \partial \Omega_{1} \cap P$. Then $\|u\|=|u|_{0}+\left|u^{\prime \prime}\right|_{0}=r$, and $|u|_{0} \leqslant r,\left|u^{\prime \prime}\right|_{0} \leqslant r$. It follows from (9) that

$$
u(t) \leqslant \frac{1}{2} t(1-t)\left|u^{\prime \prime}\right|_{0} \leqslant \frac{r}{2} t(1-t) \leqslant N t(1-t)
$$


In view of (2), (3), and (20), we have

$$
\begin{aligned}
T u(t) & =\int_{0}^{1} h(t, s) f(s, u(s)) d s \\
& \leq \int_{0}^{1} h(t, s)\left(\frac{u(s)}{s(1-s)}\right)^{\lambda} f(s, s(1-s)) d s \\
& \leq 2^{-\lambda} r^{\lambda} \int_{0}^{1} s(1-s) f(s, s(1-s)) d s
\end{aligned}
$$

and

$$
|T u|_{0} \leqslant 2^{-\lambda} r^{\lambda} \int_{0}^{1} s(1-s) f(s, s(1-s)) d s, \quad u \in \partial \Omega_{1} \cap P
$$

On the other hand,

$$
\begin{aligned}
-(T u)^{\prime \prime}(t) & =\int_{0}^{1} G(t, s) f(s, u(s)) d s \\
& \leq \int_{0}^{1} G(t, s)\left(\frac{u(s)}{s(1-s)}\right)^{\lambda} f(s, s(1-s)) d s \\
& \leq 2^{-\lambda} r^{\lambda} \int_{0}^{1} s(1-s) f(s, s(1-s)) d s
\end{aligned}
$$

and so

$$
\left|(T u)^{\prime \prime}\right|_{0} \leqslant 2^{-\lambda} r^{\lambda} \int_{0}^{1} s(1-s) f(s, s(1-s)) d s .
$$

Thus, from (21), (22), and (19),

$$
\begin{aligned}
\|T u\| & =|T u|_{0}+\left|(T u)^{\prime \prime}\right|_{0} \leqslant 2^{1-\lambda} r^{\lambda} \int_{0}^{1} s(1-s) f(s, s(1-s)) d s \\
& \leqslant r=\|u\|, \quad u \in \partial \Omega_{1} \cap P .
\end{aligned}
$$

Next, set $\Omega_{2}=\{u \in E \mid\|u\|<R\}$, where

$$
R=\max \left\{288 M, 2^{(9 \lambda+1) /(\lambda-1)}\left(\int_{1 / 4}^{3 / 4} s(1-s) f(s, s(1-s)) d s\right)^{1 /(1-\lambda)}\right\}
$$

Let $u \in \partial \Omega_{2} \cap P$. Then $\|u\|=|u|_{0}+\left|u^{\prime \prime}\right|_{0}=R,|u|_{0} \leq R,\left|u^{\prime \prime}\right|_{0} \leq R$. From (9), we have

$$
|u|_{0} \leqslant \frac{1}{8}\left|u^{\prime \prime}\right|_{0}, \quad\left|u^{\prime \prime}\right|_{0} \geqslant \frac{8}{9} R
$$


Also, by the definition of the cone $P$, we have that for $1 / 4 \leqslant t \leqslant 3 / 4$,

$$
\begin{aligned}
u(t) & =\int_{0}^{1} G(t, s)\left(-u^{\prime \prime}(s)\right) d s \geq \int_{1 / 4}^{3 / 4} G(t, s)\left(-u^{\prime \prime}(s)\right) d s \\
& \geqslant \frac{1}{4^{2}}\left|u^{\prime \prime}\right|_{0} \int_{1 / 4}^{3 / 4} G(s, s) d s \geqslant \frac{1}{2^{8}}\left|u^{\prime \prime}\right|_{0},
\end{aligned}
$$

and hence,

$$
|u|_{0} \geqslant \frac{1}{2^{8}}\left|u^{\prime \prime}\right|_{0}
$$

Since $u \in P$, from (26), we have

$$
\frac{u(s)}{s(1-s)} \geqslant 4 u(s) \geqslant|u|_{0} \geqslant \frac{1}{2^{8}}\left|u^{\prime \prime}\right|_{0}
$$

and so, from (24) and (25), for $1 / 4 \leqslant s \leqslant 3 / 4$,

$$
\frac{u(s)}{s(1-s)} \geqslant \frac{1}{2^{8}} \frac{8}{9} R \geqslant M
$$

For $1 / 4 \leqslant t \leqslant 3 / 4$, from (27) and (28), we have

$$
\begin{aligned}
T u(t) & =\int_{0}^{1} G(t, \tau) \int_{0}^{1} G(\tau, s) f(s, u(s)) d s d \tau \\
& \geqslant \int_{1 / 4}^{3 / 4} G(t, \tau) \int_{1 / 4}^{3 / 4} G(\tau, s) f(s, u(s)) d s d \tau \\
& \geqslant \frac{1}{4^{2}} \int_{1 / 4}^{3 / 4} \tau(1-\tau) d \tau \int_{1 / 4}^{3 / 4} s(1-s)\left(\frac{u(s)}{s(1-s)}\right)^{\lambda} f(s, s(1-s)) d s \\
& \geqslant \frac{1}{2^{8}}|u|_{0}^{\lambda} \int_{1 / 4}^{3 / 4} s(1-s) f(s, s(1-s)) d s .
\end{aligned}
$$

On the other hand, from (27),

$$
\begin{aligned}
-(T u)^{\prime \prime}(t) & =\int_{0}^{1} G(t, s) f(s, u(s)) d s \\
& \geqslant \int_{1 / 4}^{3 / 4} G(t, s)\left(\frac{u(s)}{s(1-s)}\right)^{\lambda} f(s, s(1-s)) d s \\
& \geqslant 2^{-8 \lambda-2}\left|u^{\prime \prime}\right|_{0}^{\lambda} \int_{1 / 4}^{3 / 4} s(1-s) f(s, s(1-s)) d s
\end{aligned}
$$


and hence,

$$
\left|(T u)^{\prime \prime}\right|_{0} \geqslant 2^{-8 \lambda-2}\left|u^{\prime \prime}\right|_{0}^{\lambda} \int_{1 / 4}^{3 / 4} s(1-s) f(s, s(1-s)) d s
$$

Now, from (29), (30), and the fact that $a^{\lambda}+b^{\lambda} \geqslant 2^{1-\lambda}(a+b)^{\lambda}$ for $\lambda \geqslant 1$ and $a$, $b>0$, we arrive at

$$
\begin{aligned}
\|T u\| & \geqslant\left(2^{-8}|u|_{0}^{\lambda}+2^{-8 \lambda-2}\left|u^{\prime \prime}\right|_{0}^{\lambda}\right) \int_{1 / 4}^{3 / 4} s(1-s) f(s, s(1-s)) d s \\
& \geqslant 2^{-8 \lambda-2}\left(|u|_{0}^{\lambda}+\left|u^{\prime \prime}\right|_{0}^{\lambda}\right) \int_{1 / 4}^{3 / 4} s(1-s) f(s, s(1-s)) d s \\
& \geqslant 2^{-9 \lambda-1}\left(|u|_{0}+\left|u^{\prime \prime}\right|_{0}\right)^{\lambda} \int_{1 / 4}^{3 / 4} s(1-s) f(s, s(1-s)) d s .
\end{aligned}
$$

Consequently, by the definition of $R$, we have

$$
\begin{aligned}
\|T u\| & =|T u|_{0}+\left|(T u)^{\prime \prime}\right|_{0} \geqslant 2^{-9 \lambda-1} R^{\lambda} \int_{1 / 4}^{3 / 4} s(1-s) f(s, s(1-s)) d s \\
& \geqslant R=\|u\|, \quad u \in \partial \Omega_{2} \cap P .
\end{aligned}
$$

Finally, from (23) and (31), by Lemma 1, the operator $T$ has at least one fixed point $u \in P \cap\left(\Omega_{2} \backslash \Omega_{1}\right)$ which is a positive $C^{2}[0,1]$ solution to the boundary value problem (1).

The proof of Theorem 1 is complete.

Proof of Theorem 2: We prove the sufficiency first. Since (5) implies (4), Theorem 1 provides a $C^{2}[0,1]$ solution $u \in P$. From $(9), u(t) \leqslant(1 / 2) t(1-t)\left|u^{\prime \prime}\right|_{0}$.

To prove that $u \in C^{3}[0,1]$, choose a positive number $c \geqslant \max \left\{M,\left|u^{\prime \prime}\right|_{0} /(2 N)\right\}$. Then, from (2) and (3), we have

$$
\begin{aligned}
\int_{0}^{1}\left|u^{(4)}(s)\right| d s & =\int_{0}^{1} f(s, u(s)) d s \leqslant c^{\mu} \int_{0}^{1} f\left(s, \frac{u(s)}{c}\right) d s \\
& \leqslant c^{\mu}\left(\frac{\left|u^{\prime \prime}\right|_{0}}{2 c}\right)^{\lambda} \int_{0}^{1} f(s, s(1-s)) d s .
\end{aligned}
$$

Now, $u^{(4)}(t)$ is absolute integrable over $[0,1]$ from (5), and hence, $u \in C^{3}[0,1]$.

To prove the necessity, let there be a positive solution $u \in C^{3}[0,1]$ of (1). The same reasoning at the beginning of the proof of Theorem 1 asserts that $u(t) \geqslant k_{1} t(1-t)$ for 
all $t \in[0,1]$ and for some constant $k_{1}>0$. Let $c \geqslant \max \left\{M, 1 /\left(k_{1} N\right)\right\}$. Then, from (2) and (3),

$$
f(t, t(1-t)) \leqslant c^{\mu} f(t,(t(1-t) u(t)) /(c u(t))) \leqslant c^{\mu-\lambda} k_{1}^{-\lambda} f(t, u(t)),
$$

and hence,

$$
\begin{aligned}
\int_{0}^{1} f(t, t(1-t)) d t & \leqslant c^{\mu-\lambda} k_{1}^{-\lambda} \int_{0}^{1} f(t, u(t)) d t=c^{\mu-\lambda} k_{1}^{-\lambda} \int_{0}^{1} u^{(4)}(t) d t \\
& =c^{\mu-\lambda} k_{1}^{-\lambda}\left[u^{\prime \prime \prime}(1)-u^{\prime \prime \prime}(0)\right]<+\infty .
\end{aligned}
$$

Thus, (5) holds and the proof of Theorem 2 is complete.

\section{REFERENCES}

[1] A.R. Aftabizadeh, 'Existence and uniqueness theorems for fourth-order boundary value problems', J. Math. Anal. Appl. 116 (1986), 415-426.

[2] R.P. Agarwal, 'On fourth-order boundary value problems arising in beam analysis', Differential Integral Equations 2 (1989), 91-110.

[3] J.R. Graef and B. Yang, 'Existence and nonexistence of positive solutions of fourth order nonlinear boundary value problem', Appl. Anal. 74 (2000), 201-214.

[4] J.R. Graef and B. Yang, 'On a nonlinear boundary value problems for fourth order equations', Appl. Anal. 72 (1999), 439-451.

[5] R. Ma and H. Wang, 'On the existence of positive solutions of fourth-order ordinary differential equations', Appl. Anal. 59 (1995), 225-231.

[6] R. Ma, J. Zhang, and S. Fu, 'The method of lower and upper solutions for fourth-order two-point boundary value problems', J. Math. Anal. Appl. 215 (1997), 415-422.

[7] Y. Yang, 'Fourth-order two-point boundary value problems', Proc. Amer.'Math. Soc. 104 (1988), 175-180.

[8] D. O'Regan, 'Solvability of some fourth (and higher) order singular boundary value problems', J. Math. Anal. Appl. 161 (1991), 78-116.

[9] Z. Wei, 'Positive solutions of singular boundary value problems of fourth order differential equations', Acta Math. Sinica 42 (1999), 715-722.

[10] S. Chen and Y. Zhang, 'Singular boundary value problem on a half-line', J. Math. Anal. Appl. 195 (1995), 449-468.

[11] D. Guo and V. Lakshmikantham, Nonlinear problems in abstract cones, Notes and Reports in Mathematics in Science and Engineering 5 (Academic Press, Inc., Boston, New York, 1988).

\footnotetext{
Department of Mathematics

Shandong University

Jinan

Shandong 250100

People's Republic of China

e-mail: szchen@ sdu.edu.cn
} 